\title{
Supramolecular Tubustecan Hydrogel as Chemotherapeutics Carrier to Improve Tumor Penetration and Local Treatment Efficacy
}

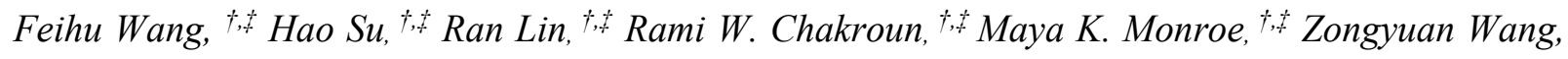

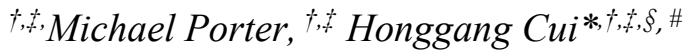

$\dagger$ Department of Chemical and Biomolecular Engineering, Whiting School of Engineering, Johns Hopkins University, Baltimore, Maryland 21218, United States.

Institute for NanoBiotechnology (INBT), Johns Hopkins University, Baltimore, Maryland 21218, United States.

$\S$ Department of Oncology and Sidney Kimmel Comprehensive Cancer Center, Johns Hopkins University School of Medicine, Baltimore, Maryland 21205, United States.

\#Center for Nanomedicine, The Wilmer Eye Institute, Johns Hopkins University School of Medicine, 400 North Broadway, Baltimore, Maryland 21231, United States.

*Corresponding author. Email: hcui6@jhu.edu 


\section{Supplementary Methods}

Materials. Amino acids and Rink Amide MBHA Resin were purchased from AAPPTEC (Louisville, KY). Camptothecin (CPT) was obtained from Ava Chem Scientific (San Antonio, TX). Doxorubicin (DOX), curcumin (Cur) L-glutathione (GSH), crystal violet, and 3-(4, 5-dimethylthiazol-2-yl)-2, 5diphenyltetrazolium bromide (MTT) were purchased from Sigma-Aldrich (St. Louis, MO). The fetal bovine serum (FBS), penicillin-streptomycin, Dulbecco's modified Eagle medium (DMEM), and 0.25\% trypsin were obtained from Gibco BRL. All other reagents and solvents were sourced through VWR.

Cell Culture. U-87 brain cancer cell line, 4T1 breast cancer cell line, and 4T1-luc cell line were generously donated by Prof. Justin Hanes at The Johns Hopkins University School of Medicine. All the cells were grown in DMEM supplemented with 10\% FBS, 100 Units/mL penicillin G sodium, and $100 \mu \mathrm{g} / \mathrm{mL}$ streptomycin sulfate. Cells were maintained at $37^{\circ} \mathrm{C}$ in a $5 \% \mathrm{CO}_{2}$ and humidified incubator. Cells grown to confluence were passaged with a 1:3 split ratio using fresh growth medium every other day after trypsinization with $0.25 \%$ trypsin-EDTA.

Mice. 6-7 week old Female NOD-scid and BALB/c mice were obtained from Charles River and kept under a $12 \mathrm{~h}$ light/dark cycle at the Animal Care Facility with food and water ad libitum. All experiments were performed in accordance with the animal protocol approved by the Animal Care and Welfare Committee at The Johns Hopkins University.

TT6 Conjugate Synthesis. The iRGD containing peptide $\mathrm{C}_{2} \mathrm{~K}$-cyl[CRGDRGPDC] was synthesized using an AAPPTEC Focus XC synthesizer via the standard Fmoc-solid phase technique. CPT-etcSSPyr was synthesized using the same method as previously reported ${ }^{1}$. ${ }_{2} \mathrm{~K}$-cyl[CRGDRGPDC] (10 mg, $7.2 \mu \mathrm{mol})$ was dissolved in an $\mathrm{N}_{2}$-purged DMSO solution of CPT-etcSS-Pyr (16 mg, $\left.28.8 \mu \mathrm{mol}\right)$ and allowed to react overnight. The solution was diluted to $9 \mathrm{~mL}$ with $0.1 \%$ aqueous TFA and purified by 
RP-HPLC. Product fractions were combined and immediately lyophilized to give TT6 as a white to yellow powder. The product identity was confirmed using ESI MS.

Transmission Electron Microscopy (TEM). All self-assembly experiments were performed at room temperature. All conjugates were directly dissolved in deionized water to make solutions of the desired concentrations. Solutions were typically aged for $24 \mathrm{~h}$ before TEM imaging. TEM samples were prepared by adding $10 \mu \mathrm{L}$ solution onto a carbon-film-coated copper grid. After careful removal of the excess liquid using a piece of filter paper, a drop of $2 \% \mathrm{w} / \mathrm{v}$ uranyl acetate aqueous solution (10 $\mu \mathrm{L}$ ) was added onto the TEM grid as a negative staining agent. Again, excess solution was removed using a piece of paper, resulting in a thin liquid film that was allowed to air dry prior to imaging. A Technai12 TWIN transmission electron microscope was used to image the prepared samples.

Circular Dichroism (CD) Spectroscopy. CD spectra were recorded on a Jasco J-710 spectropolarimeter (JASCO, Easton, MD) using a $10 \mathrm{~mm}$ path length, Spectrasil ${ }^{\circledR}$ quartz, UV-Vis absorption cell (Starna Cells Inc., Atascadero, CA). Data was normalized with respect to sample concentration and path length.

In vitro Drug Release. A $20 \mathrm{mM}$ glutathione (GSH) stock solution was prepared in $20 \mathrm{mM}$ PBS buffer (pH 7.4) while a solution of TT6 SP $(400 \mu \mathrm{M})$ was prepared in water. Equal volumes of conjugate and GSH solution were then mixed to reach a final GSH concentration of $10 \mathrm{mM}$ and TT6 concentration of $200 \mu \mathrm{M}$. The mixtures were incubated at $37^{\circ} \mathrm{C}$ for the desired period of time ( 24 hours total). Samples were taken at predetermined time points $(0,0.5,1,2,4,8,12,24 \mathrm{~h})$ and the release of CPT from the conjugate was quantified using analytical HPLC.

Cell Viability Studies. The cytotoxicity assays of TT6 SP were performed using a dose-response study. A 96-well plate was seeded with cells at a density of $5 \times 10^{3}$ cells/well and incubated at $37^{\circ} \mathrm{C}$ for 
$24 \mathrm{~h}$. The cells were then treated with varying concentrations of CPT, TT6 SP, or drug-loaded SP in cell medium and incubated for a further $48 \mathrm{~h}$. Cell viability was determined by an MTT assay. The data was fitted using the Hill equation function within the Igor Pro program to obtain the $\mathrm{IC}_{50}$ values. To test the inhibition efficacy of the hydrogels, cells were seeded in 24 -well plates at a density of $8 \times 10^{3}$ cells/well with $0.5 \mathrm{~mL}$ of DMEM. $5 \mu \mathrm{L}$ of TT6 SP, DOX/TT6 SP, or Cur/TT6 SP solution at the CPT concentration of $2 \mathrm{mM}$ was then added to a transwell insert chamber that was placed on the 24-well plate. An MTT assay was performed after incubation of the cells with the hydrogels for 1, 3, and 7 days.

Penetration in Tumor Spheroids. U-87 and $4 \mathrm{~T} 1$ cells were each seeded at a density of $1 \times 10^{4}$ cells/well in 48 -well plates that were pre-coated with $150 \mu \mathrm{L}$ of a $2 \%$ low-melting-temperature agarose. The culture plates were incubated at $37^{\circ} \mathrm{C}$ for 7 days to form spheroids. Subsequently, spheroids were treated with free CPT, TT6 SP, DOX or DOX/TT6 SP for 48 hours, rinsed three times with cold PBS, and imaged by laser scanning confocal microscopy. The fluorescence intensity of individual tumor spheroids was further determined using flow cytometry following dispersion of the tumor spheroid into single cells.

Inhibition of Tumor Spheroids. U-87 and 4T1 tumor spheroids were prepared as described above. After cell seeding for 7 days, the spheroids were incubated with $400 \mu \mathrm{L}$ DMEM containing free CPT, TT6 SP, DOX/TT6 SP, or Cur/TT6 SP at the CPT concentration of $5 \mu \mathrm{M}$. Drug-free DMEM was used as a blank control. Growth inhibition of the various drug formulations was evaluated by measuring the size of the tumor spheroids using an inverted phase microscope on days $0,1,3,5$, and 7 . Volume of the tumor spheroids was calculated using the equation $V=\left(\pi \times d_{\max } \times d_{\min }\right) / 6$, with $\mathrm{d}_{\max }$ and $\mathrm{d}_{\min }$ representing the maximum and minimum diameter of the spheroid, respectively. The volume of the 
tumor spheroid prior to treatment was set as $100 \%$ for comparison.

Invasion Assay. $5 \times 10^{4} 4 \mathrm{~T} 1$ cells were added to the upper chamber of a transwell insert coated with Matrigel (BD Biosciences). After $12 \mathrm{~h}$, the cells were treated with Cur, TT6 SP or Cur/TT6 SP in 2\% FBS culture medium, and $600 \mu \mathrm{L}$ of NIH3T3 conditioned medium was plated in the lower chamber as a chemoattractant. After $36 \mathrm{~h}$ incubation, cells in the upper wells were removed while cells in the lower wells were fixed, stained with crystal violet, and photographed using an inversion microscope.

In vivo Hydrogel Degradation. The TT6 SP solution $(8.6 \mathrm{mM}, 100 \mu \mathrm{L})$ was injected into the right backs of NOD-scid mice. The mice were photographed and sacrificed at different time intervals. The remaining hydrogel in each mouse was first photographed, and then carefully collected and diluted in water to form a solution. The content of the remaining drugs was determined using HPLC to calculate the remaining weights of the undegraded hydrogel.

\section{REFERENCES}

1. Cheetham, A. G.; Ou, Y. C.; Zhang, P. C.; Cui, H. G., Linker-Determined Drug Release Mechanism of Free Camptothecin from Self-Assembling Drug Amphiphiles. Chem. Commun. 2014, 50, 60396042. 


\section{Supplementary Figures}
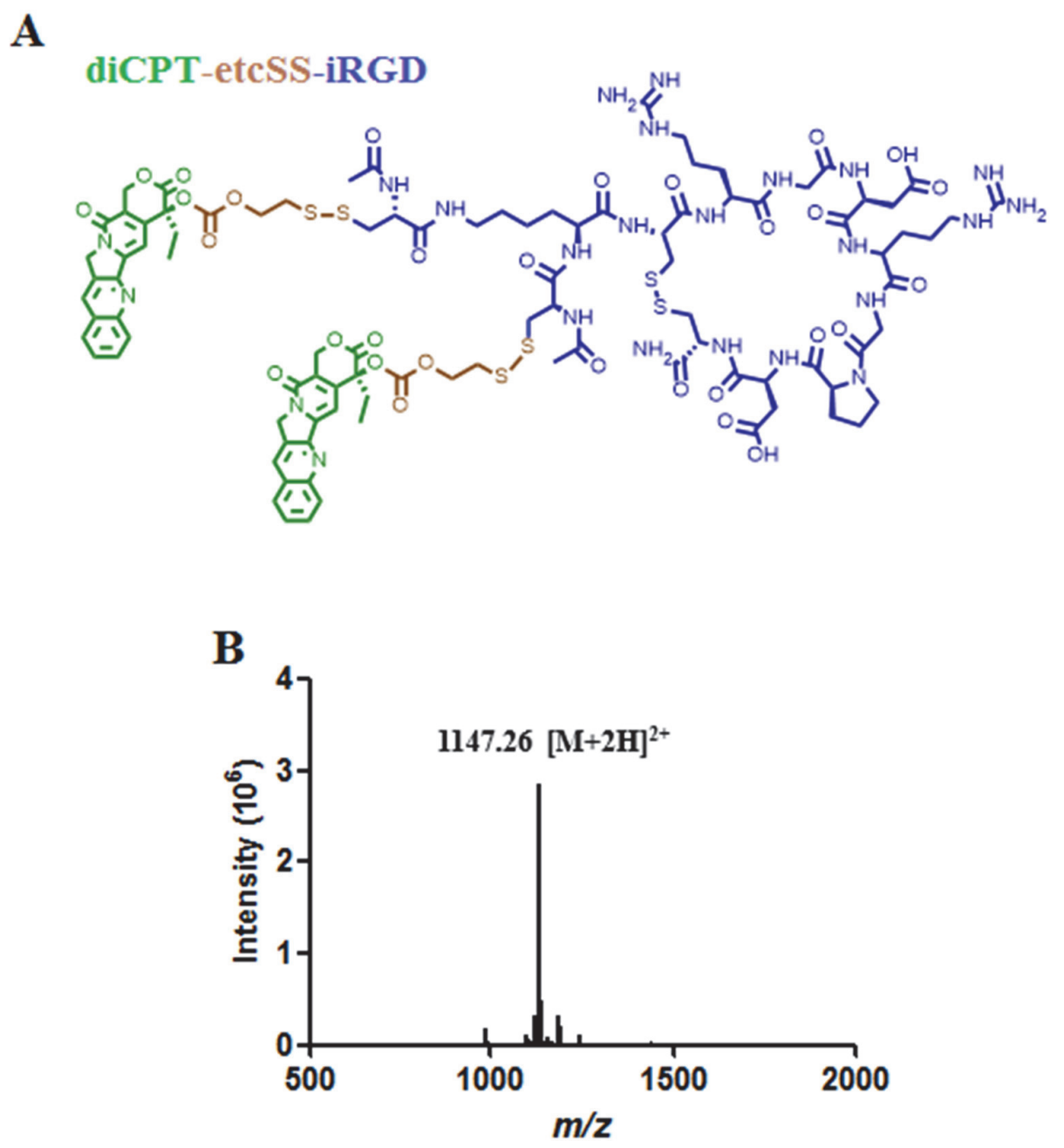

Figure S1. Characterization of the of the diCPT-iRGD amphiphile. (A) Chemical structure of the diCPT-iRGD amphiphile. (B) ESI MS profile of conjugate diCPT-iRGD showing the expected molecular weight. 

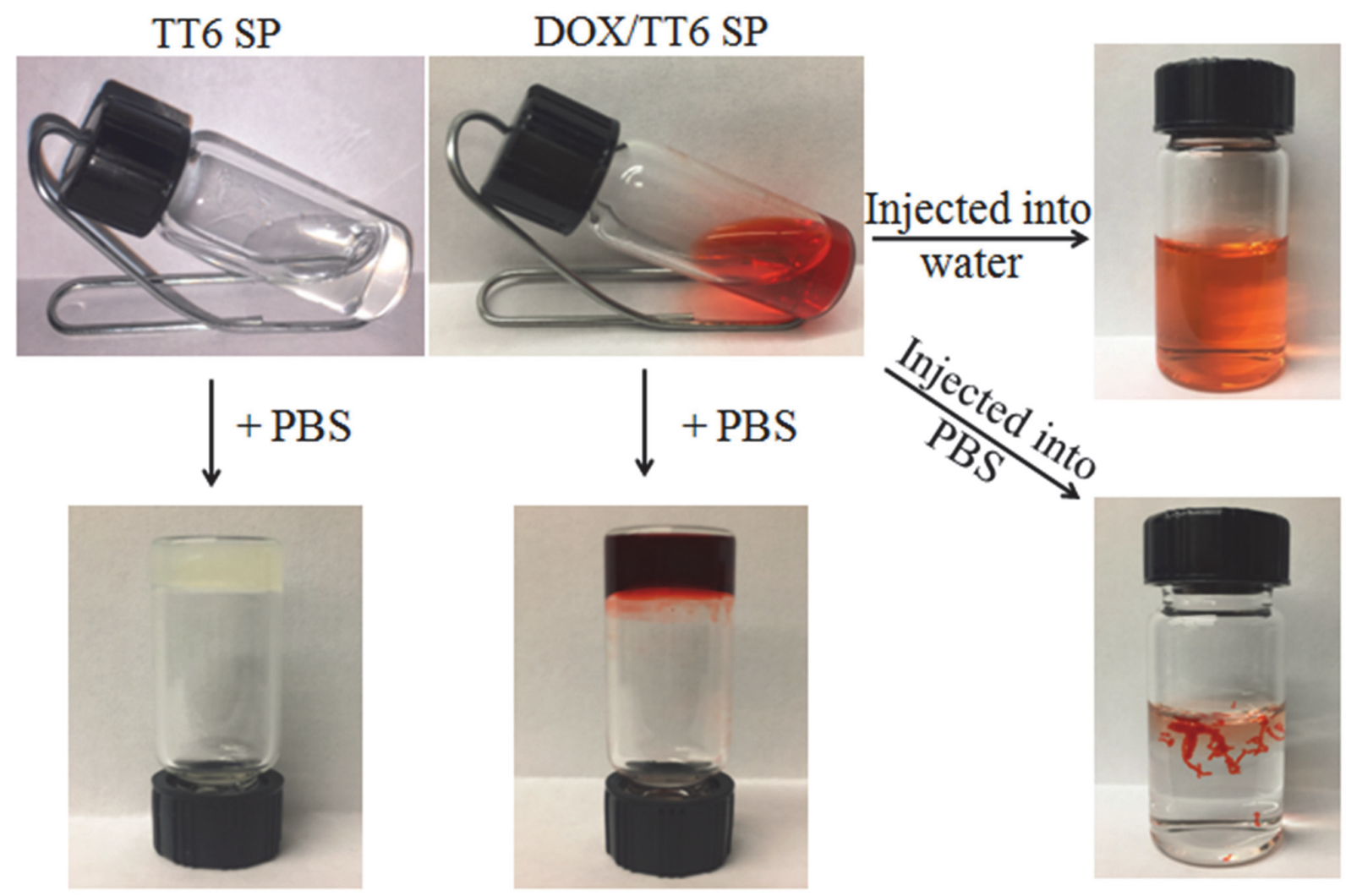

Figure S2. Hydrogel formation test of TT6 SP and DOX/TT6 SP solution following addition of PBS or injection into water or PBS. At concentrations of $2 \mathrm{mM}$ and higher, the TT6 SP and DOX/TT6 SP can be triggered to form a hydrogel immediately upon the addition of PBS. 


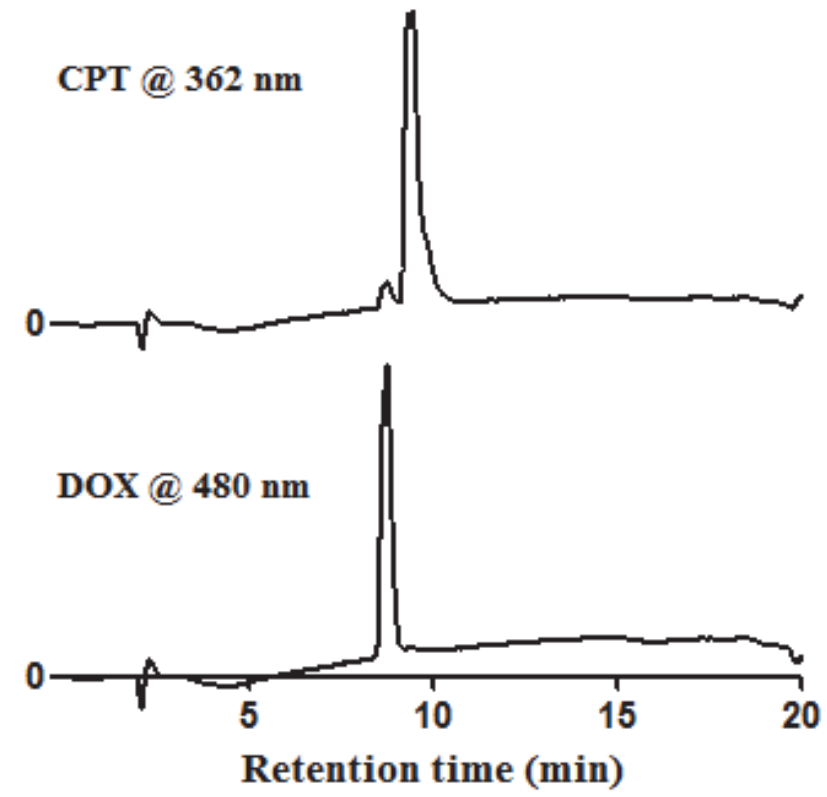

Figure S3. RP-HPLC data was used to prove TT6 SP encapsulation of DOX as evidenced by the two drug peaks at their respective detection wavelengths. DOX/TT6 SP was calculated with a DOX loading of $16.92 \mathrm{wt} \%$. 


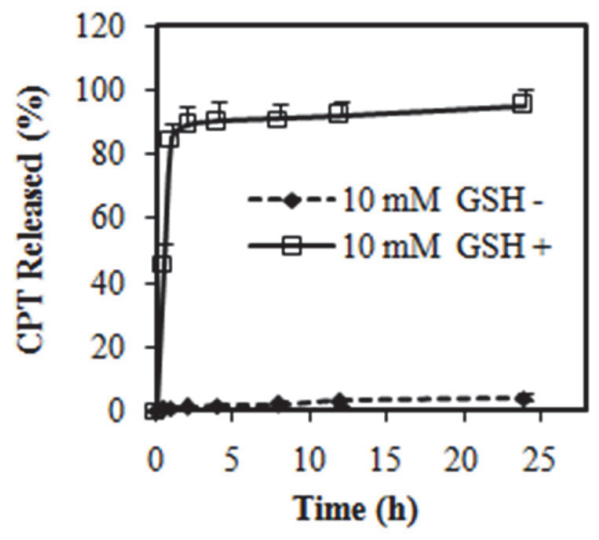

Figure S4. Release profile of CPT from TT6 SP in the presence or absence of $10 \mathrm{mM}$ GSH. In these experiments, a solution of $200 \mu \mathrm{M}$ TT6 SP was incubated in PBS buffer at $37^{\circ} \mathrm{C}$ in the presence of 10 mM GSH. GSH-induced cleavage of TT6 was observed to take place very rapidly, with $86 \%$ of CPT released within 1 hour. In contrast, TT6 concentration dropped by only 5\% over the course of 24 hours in the absence of GSH. 

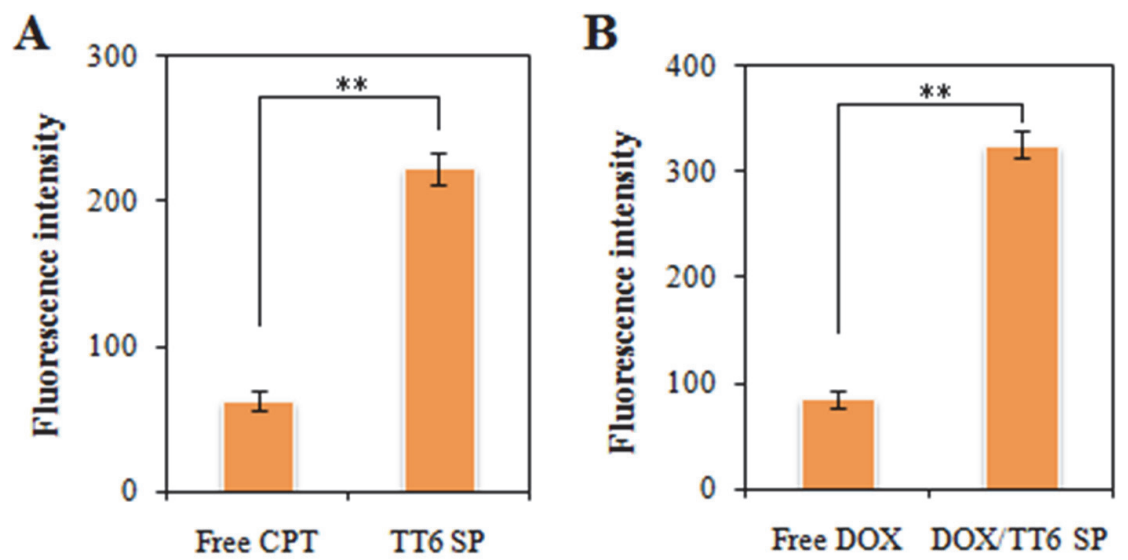

Figure S5. The fluorescent intensity of cells from U-87 spheroids after treatment with (A) CPT or TT6 SP and (B) DOX or DOX/TT6 SP for 48 hours. Fluorescence intensity displayed by tumor spheroids treated with TT6 SP and DOX/TT6 SP was significantly increased compared to those treated with free CPT and DOX. Data are expressed as mean $\pm \mathrm{SD} .{ }^{*} \mathrm{P} \leq 0.01$. 

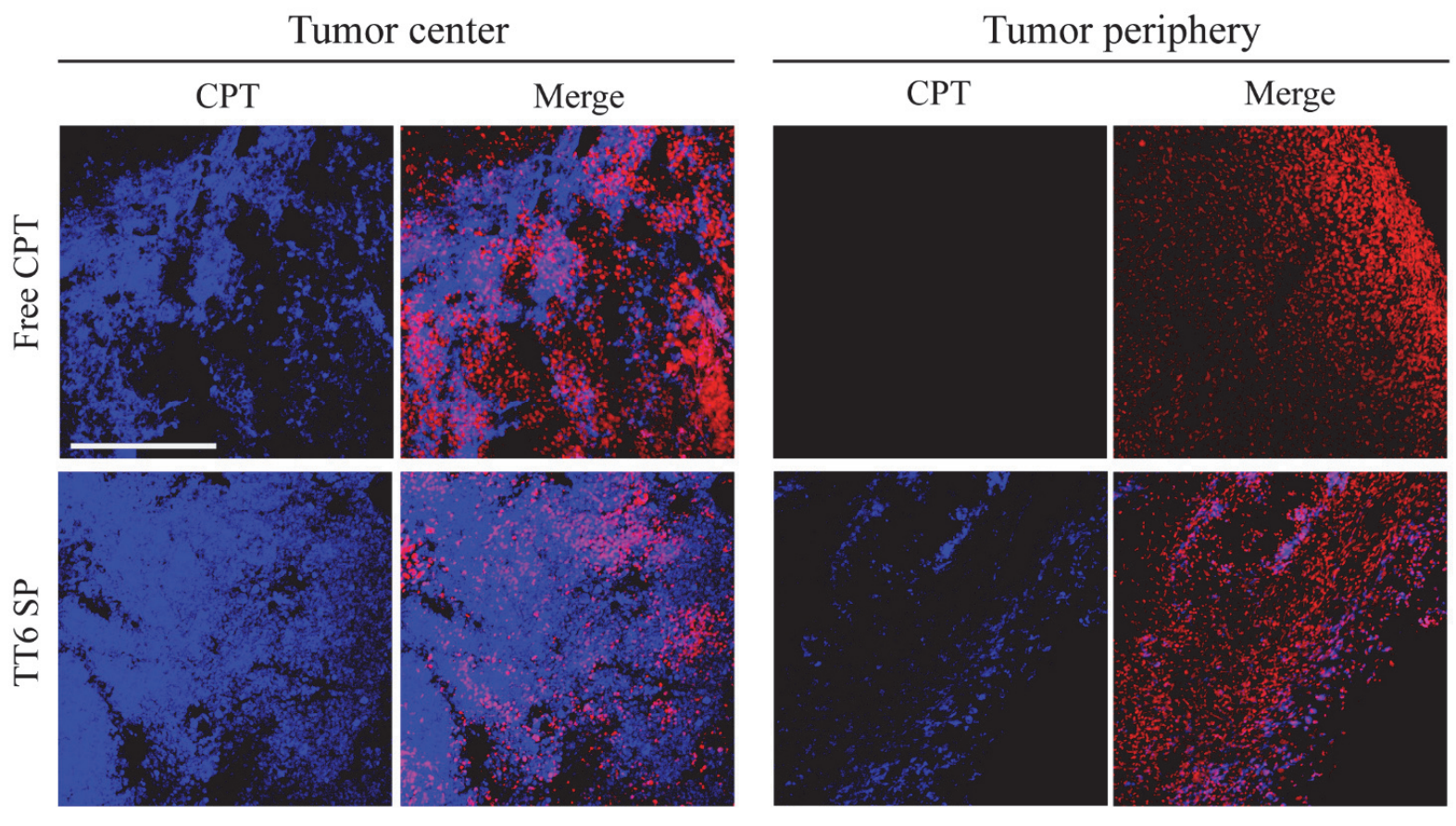

Figure S6. Fluorescence imaging of tumor sections to specifically analyze the information from tumor areas of U-87 tumor-bearing mice that locally received free CPT or TT6 SP. TT6 SP hydrogel treated tumors exhibited much stronger CPT fluorescence in the center and across the tumor, whereas for the free CPT treated mice there was nearly no fluorescence detectable at the tumor periphery. Blue: CPT, Red: DRAQ5 ${ }^{\mathrm{TM}}$ stained nuclei, scale bar $500 \mu \mathrm{m}$. 
(a) Tumor center
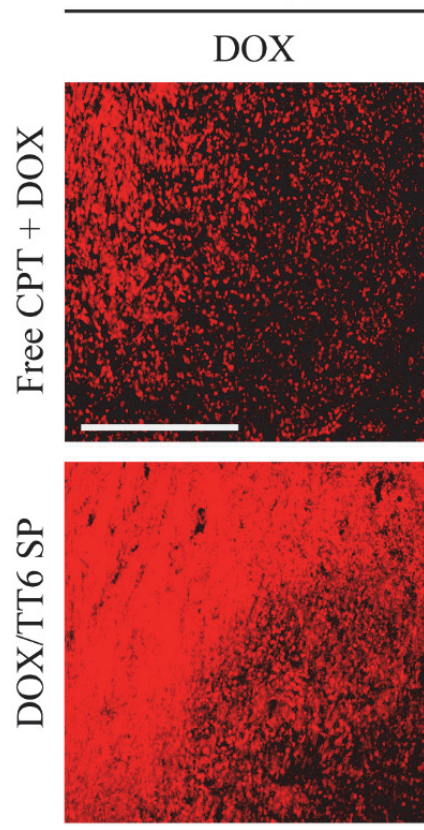

(b) Tumor periphery

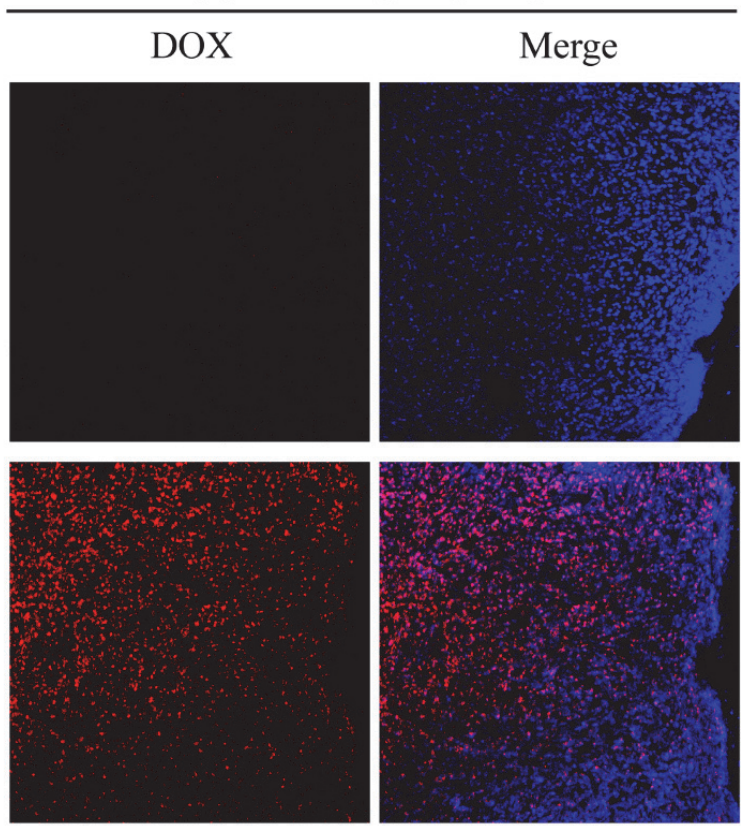

Figure S7. Fluorescence imaging of tumor sections to specifically analyze the information from tumor areas of U-87 tumor-bearing mice that locally received free CPT+ DOX or DOX/TT6 SP. DOX fluorescence was considerably low in the CPT + DOX treated tumors, whereas DOX/TT6 SP treatment led to much enhanced fluorescence in the whole tumor. Red: DOX, Blue: DAPI stained nuclei, scale bar $500 \mu \mathrm{m}$. 

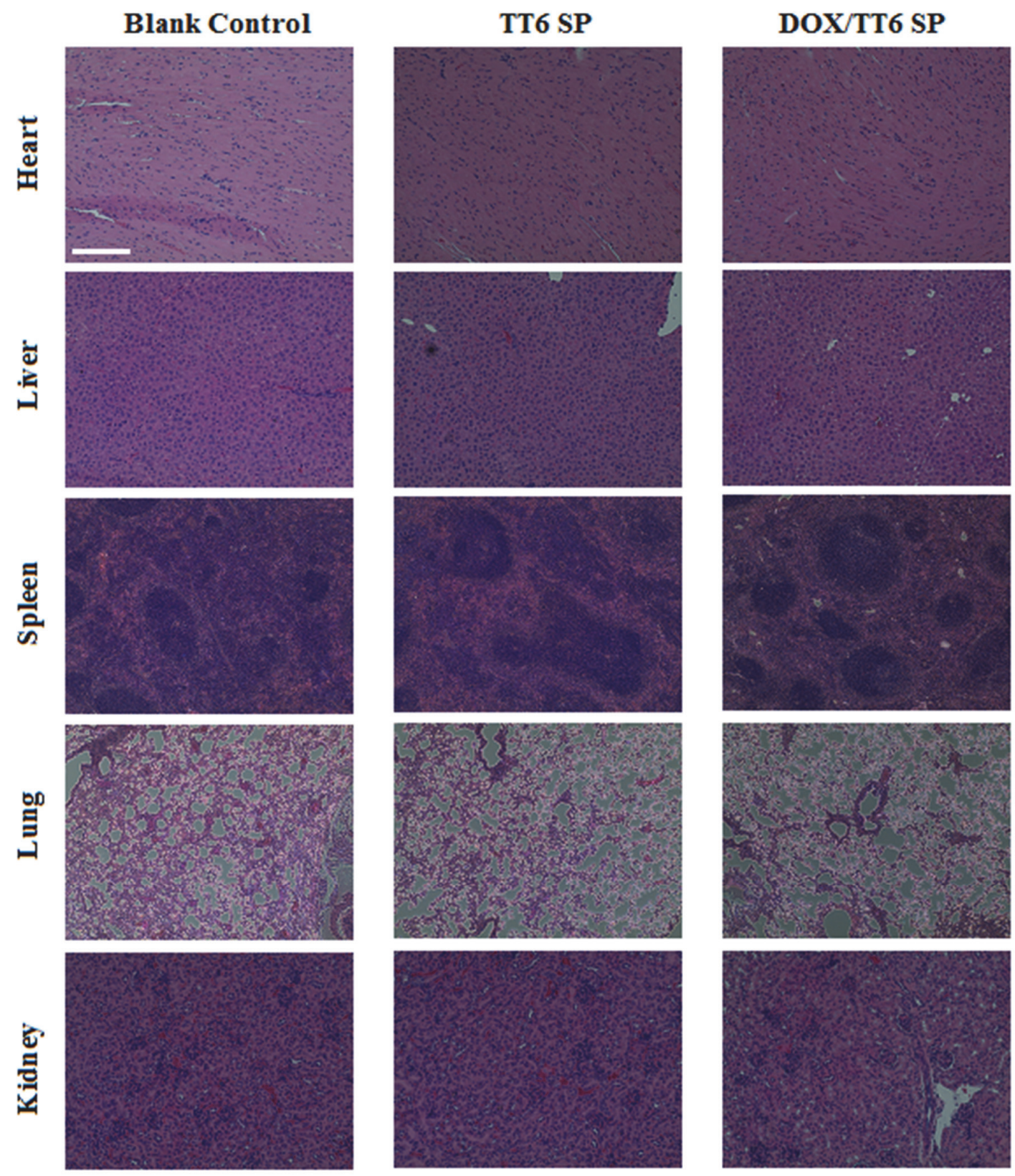

Figure S8. Histopathological examination of H\&E stained major organs from TT6 SP or DOX/TT6

SP treated mice. The organs from normal mice were set as a negative control, scale bar $500 \mu \mathrm{m}$. H\&E staining of organs obtained from the hydrogel treated groups showed no obvious pathological changes compared to those from healthy mice. 


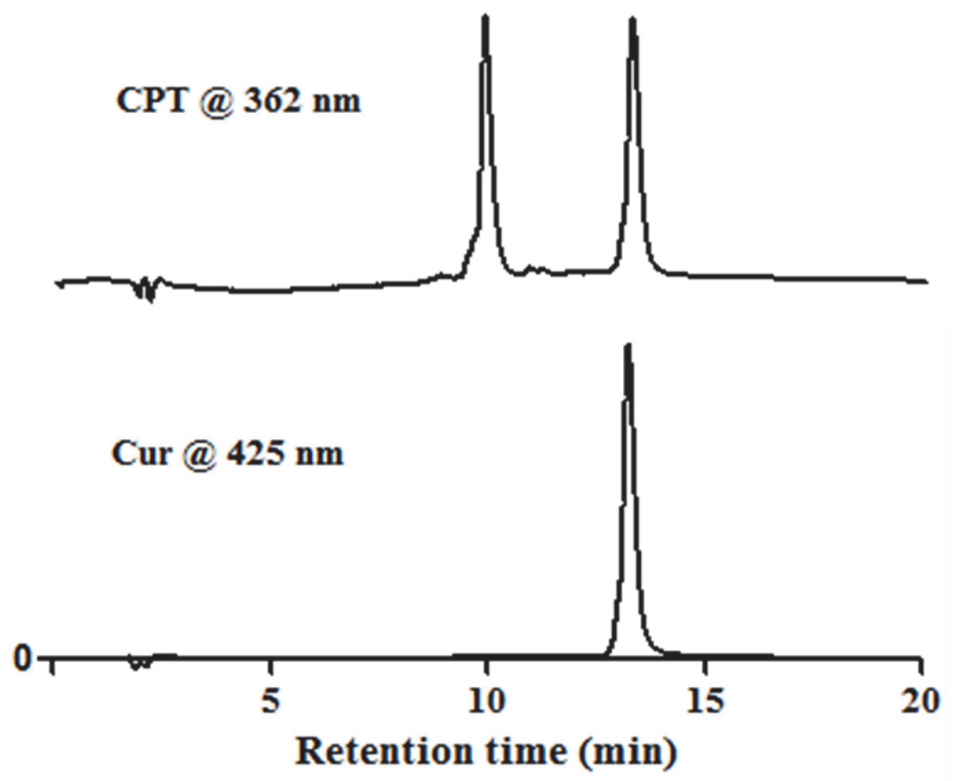

Figure S9. RP-HPLC data was used to prove TT6 SP encapsulation of Cur as evidenced by the two drug peaks at their respective detection wavelengths. TT6 SP with a Cur loading capacity of 13.3 $\mathrm{wt} \%$ was formed. 


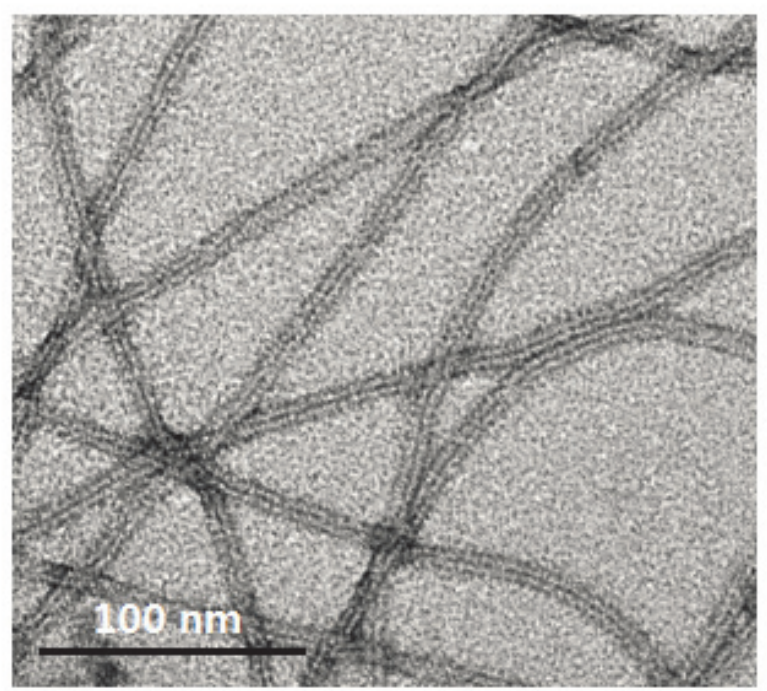

Figure S10. Representative TEM images of Cur-loaded TT6 SP. The encapsulation of Cur into TT6 SP caused an increase in nanotube diameter from $9.8 \mathrm{~nm}$ to $10.9 \mathrm{~nm}$. 

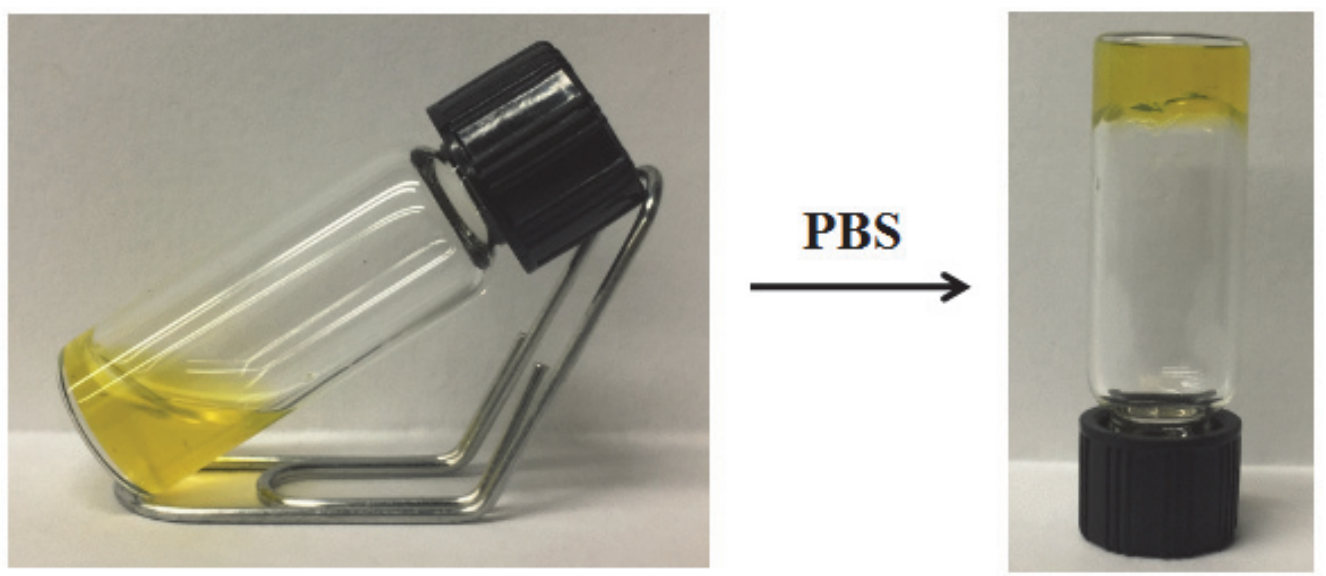

Figure S11. Hydrogel formation assay of Cur/TT6 SP solution after adding PBS. The Cur/TT6 SP still immediately formed a self-supporting hydrogel following contact with PBS solution. 

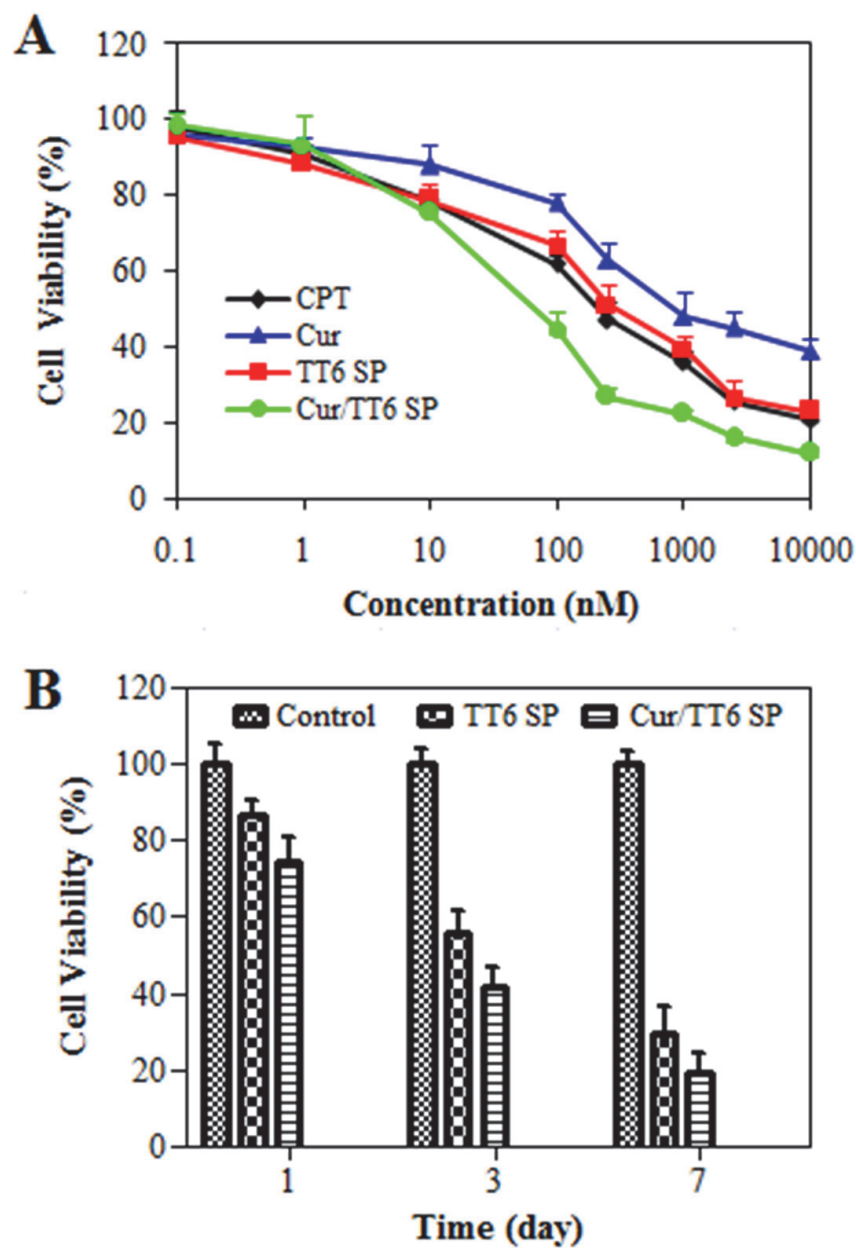

Figure S12. (A) Cytotoxicity evaluation of CPT, Cur, TT6 SP, and Cur/TT6 SP against 4T1 breast cancer cells. (B) In vitro cytotoxicity of Saline, TT6 SP gel, and Cur/TT6 SP gel towards 4T1 cancer cells. Cur/TT6 SP showed superior cytotoxicity, suggesting that the encapsulation of Cur further enhances the anti-tumor efficacy of TT6 SP. Data are given as mean \pm SD. 

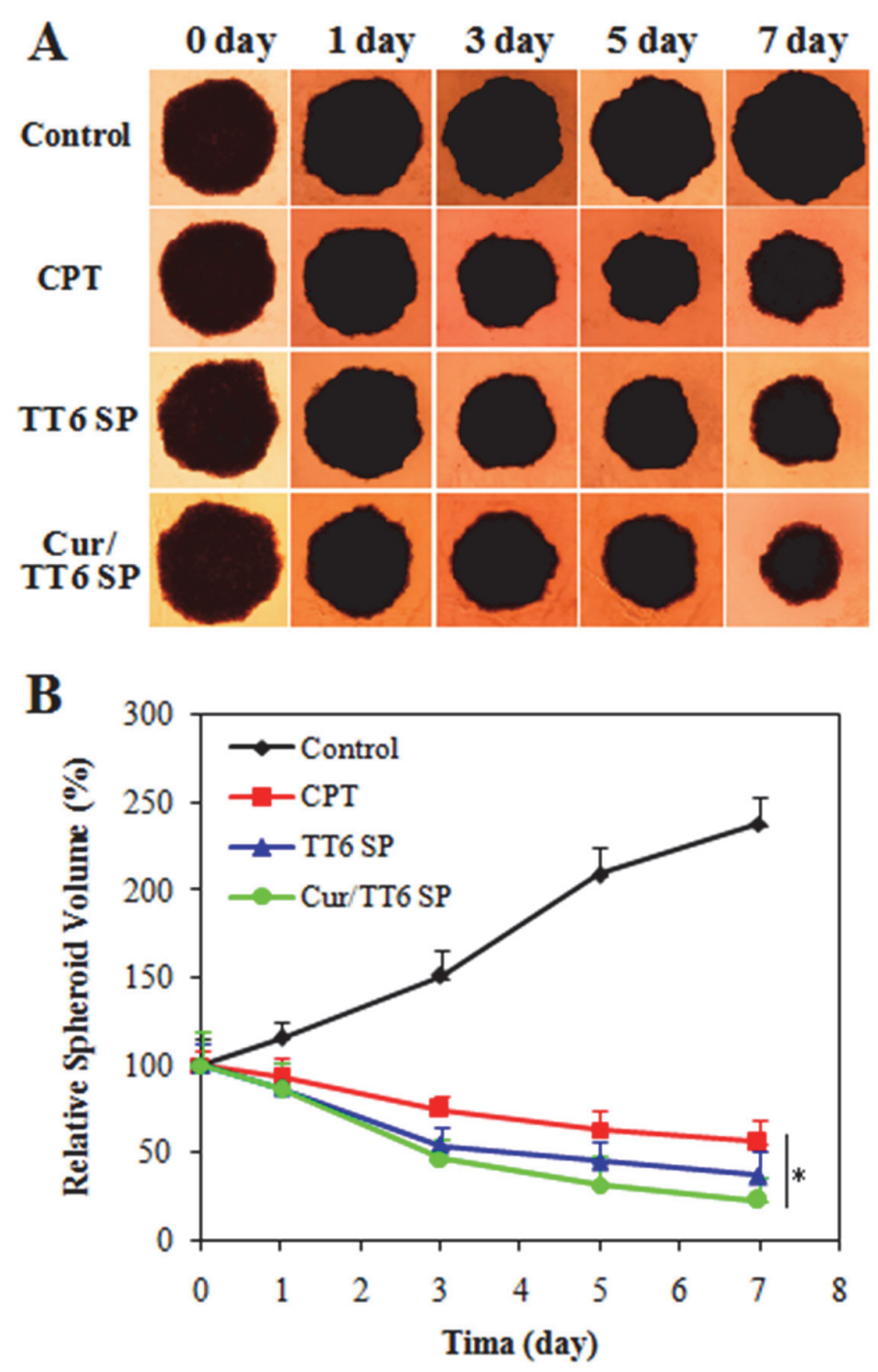

Figure S13. (A) Inhibition of tumor spheroid growth was evaluated following treatment with different drug formulations at the CPT concentration of $5 \mathrm{uM}$. Cells treated with drug-free DMEM were used as the blank control. (B) Volume of tumor spheroids treated with different formulations compared with volume at day 0 . Data are given as mean $\pm \mathrm{SD}$. 
A

Control

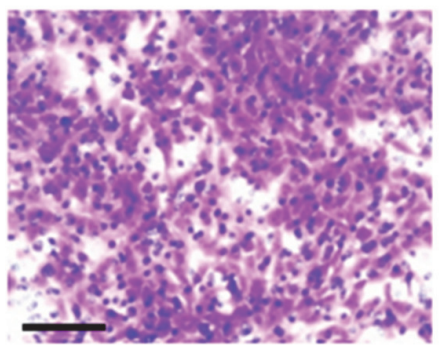

Cur

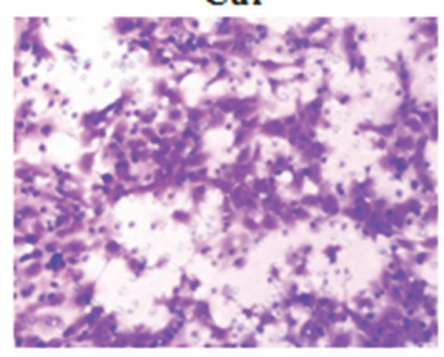

B

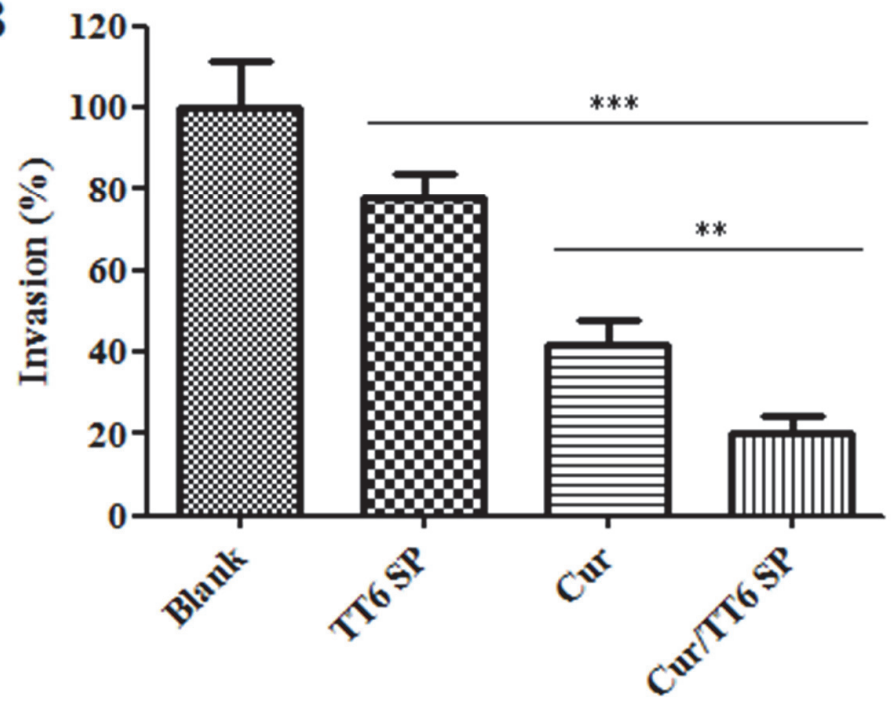

Figure S14. (A) Microscopy images of the invasion and (B) invasive percent of 4T1 cells after treatment with Cur, TT6 SP, or Cur/TT6 SP for $24 \mathrm{~h}$. Cell invasion assay exhibited that Cur/TT6 SP possess the extraordinary ability to suppress the invasion of metastatic 4T1 cells. Scale bar $200 \mu \mathrm{m}$. Data are expressed as mean $\pm \mathrm{SD} . * * \mathrm{P} \leq 0.01, * * * \mathrm{P} \leq 0.001$ 


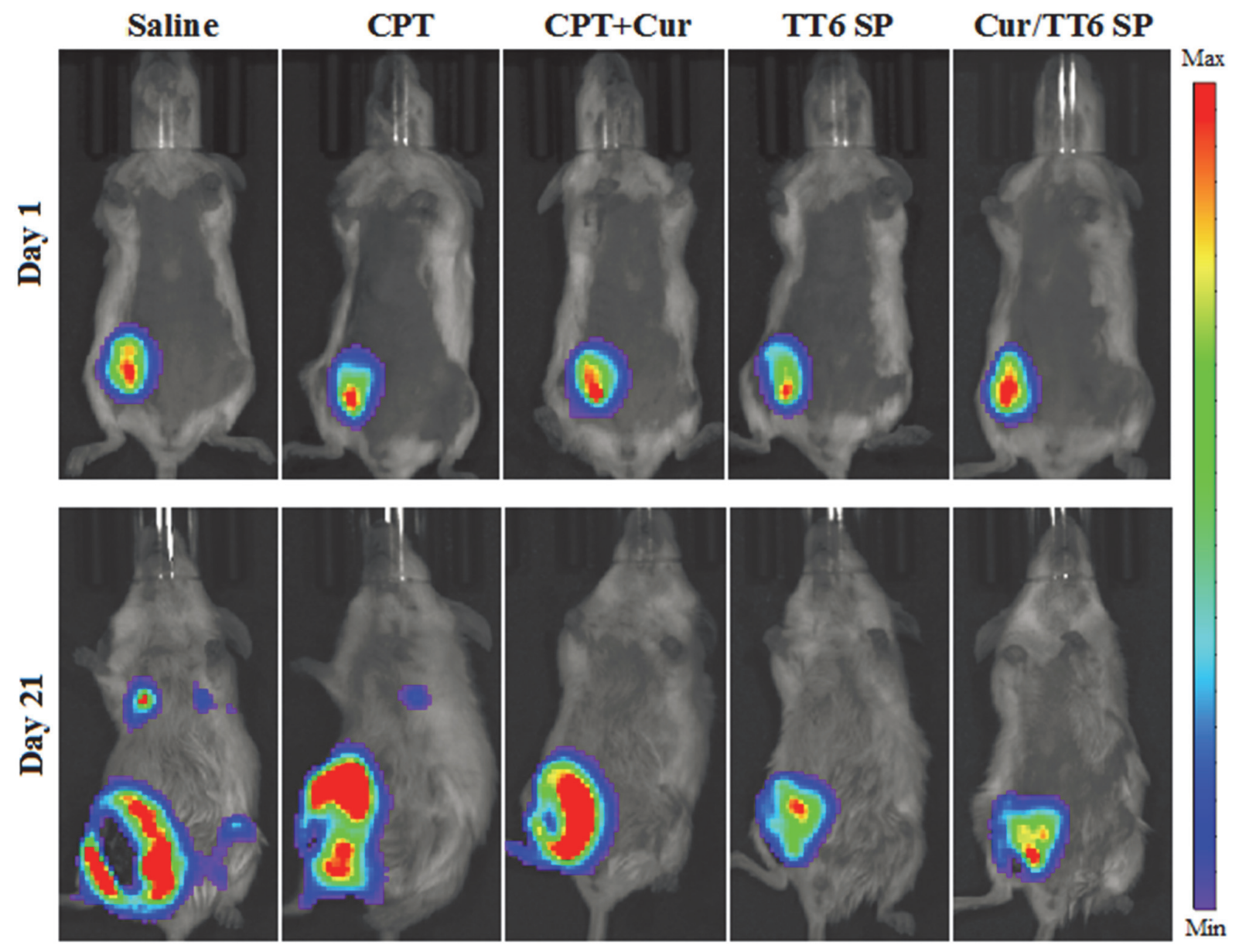

Figure S15. Representative bioluminescence images of 4T1 breast tumor bearing mice before and after different treatments. 4T1 breast cancer underwent significant pulmonary metastasis in saline treated mice. In contrast, free CPT + Cur, TT6 SP and Cur/TT6 SP therapies substantially reduced lung metastasis. 
Saline

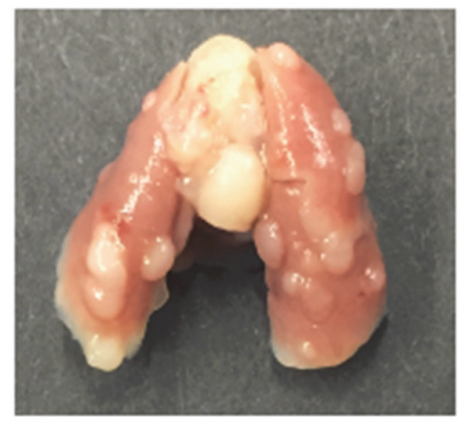

TT6 SP

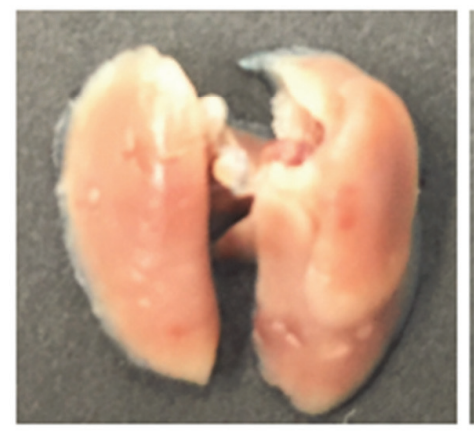

CPT

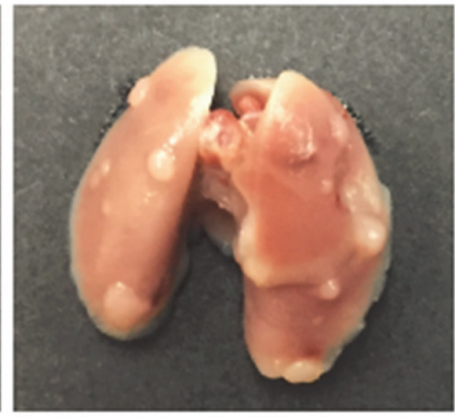

Cur/TT6 SP

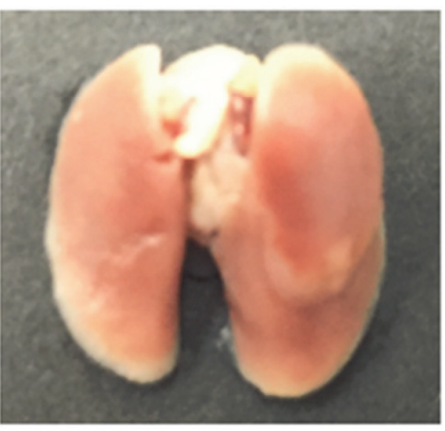

CPT + Cur

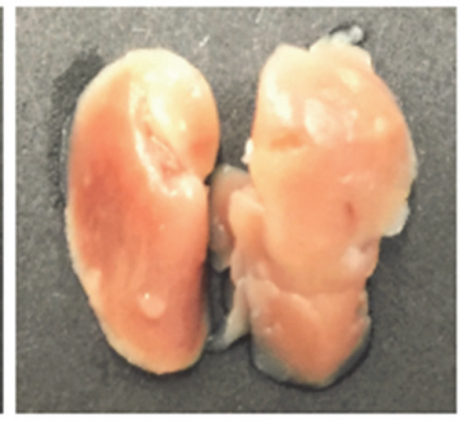

Blank

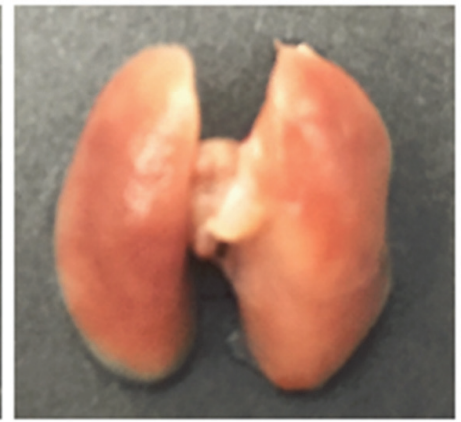

Figure S16. Representative lung images of $4 \mathrm{~T} 1$ breast tumor bearing mice on day 21 after different treatments. The Cur/TT6 SP hydrogel displayed markedly improved anti-metastatic efficacy, with no tumor metastasis visible on the lung tissue surface. 